\title{
Computer-aided high-throughput cloning of bacteria in liquid medium
}

\author{
Tuval Ben Yehezkel $^{1}$, Shiran Nagar ${ }^{1}$, Danny Mackrants ${ }^{1}$, Zipora Marx ${ }^{1}$, Gregory Linshiz ${ }^{1,2}$, Uri Shabi ${ }^{1}$, \\ and Ehud Shapiro ${ }^{1,2}$ \\ ${ }^{1}$ Department of Biological Chemistry, Weizmann Institute of Science, Rehovot, Israel and ${ }^{2}$ Department of Computer \\ Science and Applied Mathematics, Weizmann Institute of Science, Rehovot, Israel
}

BioTechniques 50:124-127 (February 2011) doi 10.2144/000113514

Keywords: Automation; cloning; robotics; high-throughput; bacteria

Supplementary material for this article is available at www.BioTechniques.com/article/113514.

Bacterial cloning was first introduced over a century ago and has since become one of the most useful procedures in biological research, perhaps paralleled in its ubiquity only by PCR and DNA sequencing. However, unlike PCR and sequencing, cloning has generally remained a manual, labor-intensive, low-throughput procedure. Here we address this issue by developing an automated, computer-aided bacterial cloning method using liquid medium that is based on the principles of $(i)$ limiting dilution of bacteria, (ii) inference of colony forming units (CFUs) based on optical density (OD) readings, and (iii) verification of monoclonality using a mixture of differently colored fluorescently labeled bacteria for transformation. We demonstrate the high-throughput utility of this method by employing it as a cloning platform for a DNA synthesis process.

Transformation followed by monoclonal culture of bacteria (i.e., cloning) is arguably the most widely used procedure in biological research. Two methods for obtaining monoclonal bacterial cultures have been established: limiting dilution of bacteria in a liquid growth medium, and plating bacteria onto solid medium in Petri dishes (1). At present, biologists have largely neglected the original dilution-based method (1) and instead routinely plate and pick colonies one-by-one from solid medium in Petri dishes. However, increasing requirements for high-throughput cloning (2-9) call for a reexamination of how monoclonal bacterial culture is obtained. Here we demonstrate that high-throughput cloning can be realized by reverting to the use of a dilution-based technique. Recent technological advancements (e.g., plate readers and liquid handling robots) enabled us to develop a high-throughput cloning methodology using liquid growth medium for standard multiwell plates that can be managed and analyzed in real time by software developed in-house, and processed using liquid-handling robots and plate readers.

\section{Materials and methods}

All of the automated procedures listed herein were executed using the Tecan
Freedom EVO 200 system (Männedorf, Switzerland) with the relevant automated peripheral equipment, including a liquid handling arm ( $\mathrm{LiHa})$, a robotic manipulator arm (RoMa), automated plate reader, centrifuge, plate shaker, and incubator. Real-time analysis and control over the processes were executed using specialized software developed in-house. Additional methods and robot control scripts using this software for the automated procedures listed herein can be found in the Supplementary Materials.

\section{Automated transformation}

Transformations were performed using an automated procedure (See script in Supplementary Materials) into Z-competent Escherichia coli (Zymo Research, Orange, CA, USA) according to manufacturer's specifications.

\section{Automated inoculations}

Cells were taken out of the plate readerincubator with real-time OD monitoring (Tecan Infinite 200 PRO series) at the predetermined $\mathrm{OD}_{600}$ value of 0.2 and diluted by a factor of $5 \times 10^{5}$ with Luria Bertani broth (LB) (see script in Supplementary Materials). Thirty-microliter inoculations were dispensed into 384-well plates containing $40 \mu \mathrm{L} \mathrm{LB} /$ well for a total volume of $70 \mu \mathrm{L} /$ well.

Automated plasmid and PCR product purification

Clones were grown overnight in $1.3 \mathrm{~mL}$ $\mathrm{LB}$ and plasmids were extracted using the QuickClean 96-Well Plasmid Miniprep Kit (Genscript, Piscataway, NJ USA). $\mathrm{PCR}$ reactions were purified using the Zymo Research DNA Clean \& Concentrator-5 kit.

Automated sequencing

Automated sequencing was performed using the BigDye Terminator v3.1 Cycle Sequencing Kit (ABI, Carlsbad, CA, USA) and sequencing reactions were purified using the 96-well Performa DTR kit (EdgeBio, Gaithersburg, MD, USA). Purified sequencing reactions were then electrophoresed on a 3730xl 96-capillary DNA Analyzer (ABI).

\section{Results and discussion}

Our high-throughput liquid-based cloning methodology is designed to be amenable to automation using standard off-the-shelf components. To this end, we programmed a Tecan Freedom EVO 200 system using 
A

$$
\text { Transformation }
$$

\section{OD monitoring}

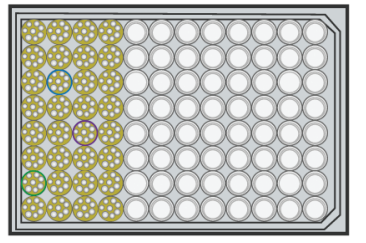

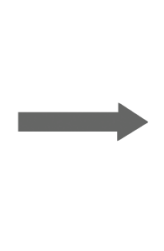

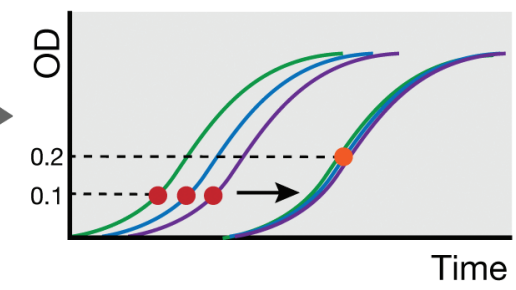

$\longrightarrow$ CFU determination

- Uniform dilution to single cells
C Cloning

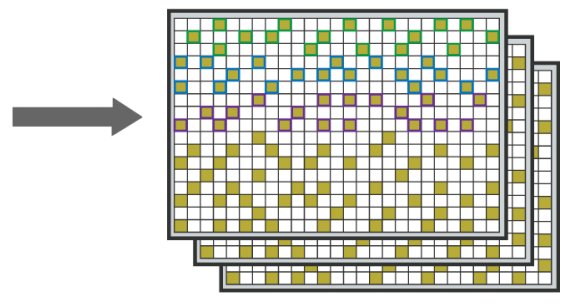

Figure 1. The five-step process for accurately performing end-to-end automated bacterial cloning in liquid. (A) Bacteria are transformed in 96 well plates (first step) (B) They are then cultured in liquid growth medium under optical density (OD) monitoring; the time it takes each transformed culture to reach the $\mathrm{OD}_{600}$ value of 0.1 is monitored in order to determine its CFU value (second step, red dots). The OD values of the cultures are then synchronized by the appropriate dilutions (third step, black arrow). The synchronized cultures are grown to OD $=0.2$ (fourth step, orange dot). (C) At OD $=0.2$ they are immediately cloned to 384-well plates using a predefined dilution with liquid growth medium (fifth step). The cultures plated in the 384-well plates produce a ratio of one positive well for every three negative wells, thereby assuring that the positive wells are monoclonal.

in-house robot control software to carry out the method. Automated procedures were developed for the entire process including ligation, transformation, clonal amplification, plasmid purification and DNA sequencing. A general overview of the method, without specification of robotics or software parts, is depicted in Figure 1.

High-throughput cloning of

bacteria in liquid medium

For automated bacterial cloning, competent bacteria not requiring heat shock or recovery were transformed in 96-well plates using an automated procedure. Following transformation, all cultures were diluted 1:860 into transparent 96-well plates in LB-Amp so that their $\mathrm{OD}_{600}$ measurement $(0.075 \pm$ 0.005 using $300 \mu \mathrm{L}$ LB-Amp aliquots) was not masked by the absorbance of untransformed dead cells.

Diluted transformations were then cultured at $37^{\circ} \mathrm{C}$ in a plate reader with automated real-time $\mathrm{OD}_{600}$ monitoring (with automatic subtraction of the background $\mathrm{OD}_{600}$ reading of 0.075 ). The $\mathrm{CFU}$ of each transformation was determined based on timing each culture's growth to the $\mathrm{OD}_{600}$ value of 0.1 (see "Analog CFU inference" section, and Supplementary Materials). After CFU determination, if it appeared that the ODs of all the transformed cultures were not synchronized (due to different CFUs), they were then synchronized using an automated dilution-based procedure. Based on the OD readings, robotic scripts were automatically generated using in-house software to equalize ODs by the appropriate dilutions. Once the robot executed the scripts, the cultures were placed back in the plate reader to verify that the ODs were synchronized. IfOD readings indicated that they were still not synchronized, the procedure was repeated. This synchronization step allows for the use of a uniform dilution factor for all transformations in the single-cell inoculation step.

The synchronized transformations were cultured until they reached an $\mathrm{OD}_{600}$ of $0.2 \pm 0.02$ (which equals $4 \times 10^{6}$ cells $/ \mathrm{mL}$ ), whereupon they were all diluted by a factor of $5 \times 10^{5}$ to obtain a concentration of 8 viable transformed cells per $\mathrm{mL}$. Inoculation of 30- $\mu \mathrm{L}$ aliquots into 384-well plates resulted in $25 \%$ of wells containing a single cell. In principle, other OD values still within the range of linear correlation to cell density can also be used, albeit with a different dilution factor. Once grown overnight, the 384-well plates displayed a predefined ratio of positive (growth) to negative (no growth) wells of $\sim 1: 3$, as determined by a plate reader OD scan. The dilution that resulted in $\sim 25 \%$ of wells containing single cells from $\mathrm{OD}_{600}=0.2$ was determined beforehand in pilot experiments (See Supplementary Material, "Description of pilot experiments to determine the dilution to achieve single cells" section and spreadsheet). Diluting to single cells using this method is independent of transformation efficiency (See Supplementary Figure 5).

\section{Verification of clonality us-}

ing DNA sequencing

Culture clonality in individual wells was accomplished through a combination of an appropriately low positive to negative well ratio, as well as through the optional use of a mixture of fluorescently labeled bacteria of different colors during transformation, as described in the "Verification of monoclonality using fluorescent bacteria" section. The maximum number of monoclonal wells was obtained when the average number of viable cells/aliquot is 1 ; however, this does not minimize the number of polyclonal wells. The cost and effort of sequencing false positive (i.e., polyclonal) colonies outweighs that of plating more negative wells, and therefore we aim for a low ratio of positive: negative wells. We chose a ratio of 1:3, which statistically favors the monoclonality of the positive wells. Nevertheless, since we could not rule out possible false positives caused by clumping of bacteria or contamination, the frequency of polyclonal wells was determined by DNA sequencing.

Using our automated liquid cloning method, we cloned a synthetic DNA library of a 768-bp fragment that was mutated at a high frequency. Previous comprehensive sequencing analysis showed that clones from this library have an average of $4-5$ mutations randomly positioned along the 768-bp sequence (10). As a result, this library is, in effect, barcoded, with each clone having a unique mutational pattern. Monoclonal and polyclonal cultures from this cloned library are easily distinguishable using DNA sequencing, since polyclonal cultures always harbor more than a single plasmid sequence due to the high error-rate of the library (See Figure 2B). Four positive wells from our cloning procedure were each manually plated onto four separate Petri dishes, and three colonies from each Petri dish were manually picked and sequenced. Sequence analysis showed that colonies picked from the same Petri dish (i.e., inoculated with cells from a single positive well) reproducibly propagated plasmids with the exact same pattern of mutations. Conversely, groups of colonies picked from different Petri dishes (i.e., those groups inoculated from different positive wells) reproducibly propagated plasmids exhibiting a completely different pattern of mutations from each other. These results attest to the monoclonality 
A
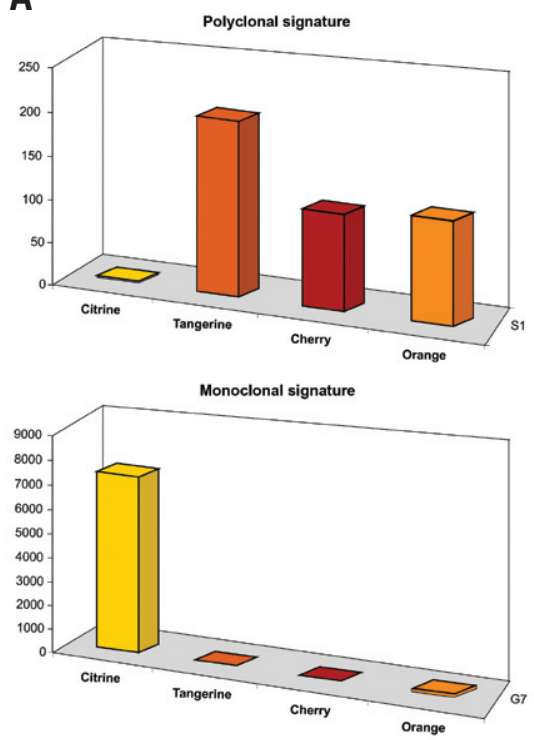

Figure 2. Clonal verification. (A) Examples of polyclonal (top) and monoclonal (bottom) fluorescent signatures. Each well of a 384-well plate after cloning into bacteria expressing one of four fluorescent proteins is subjected to the appropriate fluorescence measurement for all four proteins. Wells of monoclonal origin (bottom) display an emission signature only for one of the proteins. Polyclonal wells (top) display emission signatures for more than one protein. We do not attempt to decipher which fluorescent proteins are present in polyclonal cultures since these are false-positive cultures which are not useful for cloning purposes. In principle, this can be done by using a more sophisticated analysis of the sequencing results and/or the fluorescence emission, but is not within the scope of this method. (B) Examples of clonality verification using DNA sequencing of barcoded DNA. DNA sequences of polyclonal cultures (top electropherogram) are easily distinguishable from monoclonal cultures (bottom electropherogram) due to insertions, deletions, and substitutions. (C) The theoretical probability for obtaining true (monoclonal) clones is maximized when cells are plated at an average concentration of 1 cell/well (blue plot). Nevertheless, at this concentration there is a considerable probability of wells being polyclonal (red plot, $25 \%$ ). By using fluorescent detection the probability of false positive (polyclonal) wells is reduced considerably (black plot) and the optimal concentration ( 1 cell/well) can be used. Alternatively, a lower average number of cells per well can be used at the price of more negative (no growth) wells. Note that all three curves represent computed theoretical probabilities.

of the positive wells and exclude falsepositive results caused by cell aggregates or contamination. Therefore, false-positive clones were not produced at any significant rate when a low ratio of positive to negative wells was used.

\section{Verification of monoclonality}

using fluorescent bacteria

For additional verification of clonality, we developed a method using fluorescent bacteria to determine whether a positive well is monoclonal or polyclonal. We used a competent cell mixture containing four bacteria, each expressing a different fluorescent protein. Polyclonal cultures should have a mixed fluorescence signature resulting from the fluorescence of differently colored bacteria. In contrast, cultures of monoclonal origin should have a single fluorescence signature corresponding to the founding cell of the culture. To this end, four bacterial lines, each expressing a different fluorescent protein (Cherry, Citrine, Orange, and Tangerine.), were established in E. coli. The fluorescence signature of each of the bacteria was obtained separately (See Supplementary Figure S3A). Monoclonal cultures generated by our cloning method were identified by comparing their fluorescence signature with all four reference signatures and looking for a perfect match in terms of emission pattern and intensity.

We transformed our synthetic DNA library into a mixture of the four different fluorescent protein-expressing competent cells and generated cloned cultures using a dilution factor that would generate roughly equal numbers of monoclonal and polyclonal cultures. We measured the fluorescence signatures of 27 cultures and determined whether their signatures were identical to one of the monoclonal reference signatures. If not, they were considered to be polyclonal. These clones were then sequenced and the predictions were shown to be correct (See Supplementary Figure S3B for examples). As shown in Supplementary Figure S3A, there is a certain degree of overlap between the fluorescence spectra of some of these four fluorescent proteins. In order to distinguish between monoclonal and polyclonal cultures, the emission of each culture is recorded in four wavelengths, corresponding to one of the four proteins (as in the reference culture signatures). This fluorescence signature is then compared with the signal obtained from each of the four reference monoclonal cultures (Supplementary Figure S3A) in terms of emission pattern and intensity. If the queried culture is monoclonal (Supplementary Figure S3B), then its fluorescence signature (i.e., its emission pattern and intensity at all four wavelengths) will be identical to that of one of the four reference cultures (Supplementary Figure S3A). If not, then the culture is considered to be polyclonal (13 out of the 27 examined; see Supplementary Figure S3B). In the future, this comparison may be performed automatically. While we used a mixture of four different fluorescent reporter bacteria, this number can be further expanded with bacteria expressing other fluorescent proteins (11) if a higher degree of certainty is required. Using fluorescent bacteria enabled us to inoculate single cells with higher densities in multi-well plates without having to process polyclonal cultures (Figure 2C).

\section{Analog CFU inference}

Analog CFU inference is used to evaluate the number of independent transformants in the initial transformation (CFU), evaluate the quality and complexity of the library, and to estimate the number of independent clones that need to be screened. Determining CFU from Petri dishes with high throughput is an exhaustive task $(7,12,13)$, and may be largely relieved using analog CFU inference, which infers CFU based on the time it takes a transformed culture to reach the predetermined OD of 0.1 when compared with a reference standard curve (Figure 3). For example, a culture starting from $10^{3}$ transformed cells $\left(\mathrm{CFU}=10^{3}\right)$ will reach an OD of 0.1 exactly one doubling time slower than a culture starting from $2 \times 10^{3}$ cells (CFU $=2 \times 10^{3}$ ) and so on. We find the correlation between time-to-OD and CFU to be valid across the practical range of CFU values (Figure 3). Additionally, it allows CFUs to be obtained for 96 or 384 transformations in parallel using a plate reader. Although intended for standard cloning use, cloned DNA may be toxic and can alter growth rate compared with standard $E$. coli strains used in most labs. In these cases, a dedicated standard curve for that particular 


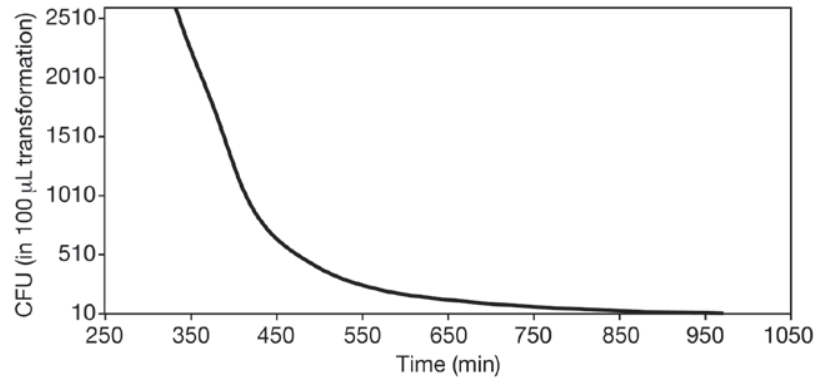

Figure 3. CFU correlates to time-to-0D. A standard curve that describes the CFU value of a transformed culture as a function of the time it takes it to reach the predefined $\mathrm{OD}_{600}$ value of 0.1 . This establishes an accurate correlation between liquid CFU and time-to-OD $=0.1$ and is used to rapidly determine the CFU value in high-throughput without colony counting.

strain may be made. See the Supplementary Materials for additional results and descriptions regarding the standard curve. Analog CFU inference may also be beneficial in a clinical setting since it provides CFU data more quickly and with higher throughput compared with colony counting on Petri dishes.

Computer-aided cloning and sequencing of a DNA library

In order to demonstrate the utility of our cloning method in high-throughput research, we cloned and sequenced our DNA library of synthetic bar-coded 768-bp fragments. We performed 32 automated transformations. Each transformation was of a diverse population of DNA molecules from which many clones could be made and sequenced. We carried out our automated cloning procedure and 24 aliquots from each diluted transformation were inoculated into 768 wells (two separate 384-well plates) with a positive to negative well ratio of 1:3. Liquid colonies were automatically picked and expanded by growth in $2 \mathrm{~mL} \mathrm{LB}$ plus antibiotic overnight. Plasmid DNA was extracted using an automated procedure from a random sample of 96 positive wells (three from each of the 32 transformations) and then sequenced using an automated procedure. In this example, we chose not to clone or sequence with higher throughput (thousands-scale) since it is not necessary for illustrative purposes. The sequencing results of the bar-coded library show that positive wells were indeed monoclonal: only two wells out of the 96 sequenced propagated more than one plasmid, while the rest propagated only one. These two wells likely reflect the natural rate of double plasmid transformation (14). Throughput in the hundreds to thousands (sequenced clones) scale per batch can also be accomplished by starting with more transformations and/or plating more 384-well plates.
A potential drawback of this method is that the representation of clones in the final library may be distorted by different growth rates for toxic or slow-growing clones. However, this may not be a major issue if the clones are not grown in strains or under conditions that induce expression of the cloned genes.

Traditional cloning into Petri dishes is difficult to upscale and automate for the average lab. Although bacterial cloning by dilution was neither originally intended nor applicable for high-throughput applications, we found that the basic principles of cloning by dilution to be better suited to the requirements of automation given the technology available today.

One of the major obstacles facing the development of future cloning platforms with higher throughput capabilities than those demonstrated herein (similar to the throughput of next-generation sequencing) is the ability to individually physically address each clone. Once such platforms become available, then bacterial cloning could potentially advance even beyond the current limit.

\section{Acknowledgments}

We thank Amit Mishali for the prompt and excellent preparation and design of figures. This research was supported by The Converging Technologies Program (grant no. 1694/07) of The Israel Science Foundation, The European Union and Paul Sparr Foundations. E.S. is the incumbent of the Harry Weinrebe Professorial Chair of Computer Science and Biology.

\section{Competing interests}

A patent is being filed by Yeda Ltd., the licensing art of the Weizmann Institute of Science, on the work described by this manuscript. FP7-ERC-AdG, Miel de Botton Aynsley,

\section{References}

1. Weiss, R.A. 2005. Robert Koch: the grandfather of cloning? Cell 123:539-542.

2. Kitagawa, M., T. Ara, M. Arifuzzaman, T. Ioka-Nakamichi, E. Inamoto, $\mathrm{H}$. Toyonaga, and H. Mori. 2005. Complete set of ORF clones of Escherichia coli ASKA library: Unique Resources for Biological Research. DNA Res. 12:291-299.

3.Baba, T., T. Ara, M. Hasegawa, Y. Takai, Y. Okumura, M. Baba, K.A. Datsenko, M. Tomita, et al. 2006. Construction of Escherichia coli K-12 in-frame, single-gene knockout mutants: the Keio collection. Mol. Syst. Biol. 2:2006.0008.

4.Zaslaver, A., A. Bren, M. Ronen, S. Itzkovitz, I. Kikoin, S. Shavit, W. Liebermeister, M.G. Surette, and U. Alon. 2006. A comprehensive library of fluorescent transcriptional reporters for Escherichia coli. Nat. Methods 3:623-628.

5. Dieckman, L., M. Gu, L. Stols, M.I. Donnelly, and F.R. Collart. 2002. High throughput methods for gene cloning and expression. Protein Expr. Purif. 25:1-7.

6. Cox, J.C., J. Lape, M.A. Sayed, and H.W. Hellinga. 2007. Protein fabrication automation. Protein Sci. 16:379-390.

7. Uber, D.C., J.M. Jaklevic, E.H. Theil, A. Lishanskaya, and M.R. McNeely. 1991. Application of robotics and image processing to automated colony picking and arraying. BioTechniques 11:642-647.

8. Ben Yehezkel, T., G. Linshiz, H. Buaron, S. Kaplan, U. Shabi, and E. Shapiro. 2008. De novo DNA synthesis using single molecule PCR. Nucleic Acids Res. 36:e107.

9.Betton, J.-M. 2004. High throughput cloning and expression strategies for protein production. Biochimie 86:601-605.

10. Linshiz, G., T.B. Yehezkel, S. Kaplan, I. Gronau, S. Ravid, R. Adar, and E. Shapiro. 2008. Recursive construction of perfect DNA molecules from imperfect oligonucleotides. Mol. Syst. Biol. 4:191.

11. Shaner, N.C., P.A. Steinbach, and R.Y. Tsien. 2005. A guide to choosing fluorescent proteins. Nat. Methods 2:905-909.

12.Breed, R.S. and W.D. Dotterrer. 1916. The number of colonies allowable on satisfactory agar plates. J. Bacteriol. 1:321-331.

13. Tomasiewicz, D.M., D.K. Hotchkiss, G.W. Reinbold, R.B. Read, Jr., and P.A. Hartman. 1980. The most suitable number of colonies on plates for counting. J. Food Prot. 43:282-286.

14. Weston, A., G.O. Humphreys, M.G. Brown, and J.R. Saunders. 1979. Simultaneous transformation of Escherichia coli by pairs of compatible and incompatible plasmid DNA molecules. Mol. Gen. Genet. 172:113-118.

Received 8 April 2010; accepted 29 December 2010.

Address correspondence to Ehud Shapiro, Department of Computer Science and Applied Mathematics, Weizmann Institute of Science, Rehovot, 76100, Israel. e-mail: ehud.shapiro@ weizmann.ac.il

To purchase reprints of this article, contact: carmelitag@fosterprinting.com 